\title{
Pharmacogenomics/Genetics Methods and Supporting Information Supplemental Qualifiers Dataset
}

National Cancer Institute

\section{Source}

National Cancer Institute. Pharmacogenomics/Genetics Methods and Supporting

Information Supplemental Qualifiers Dataset. NCI Thesaurus. Code C147236.

A dataset containing supplemental information, specifically non-standard variables, to parent records in the pharmacogenomics/genetics methods and supporting information domain. 